\title{
INTERVIEW
}

For reprint orders, please contact: reprints@futuremedicine.com

\section{Neuropathic pain: research, treatment and education}

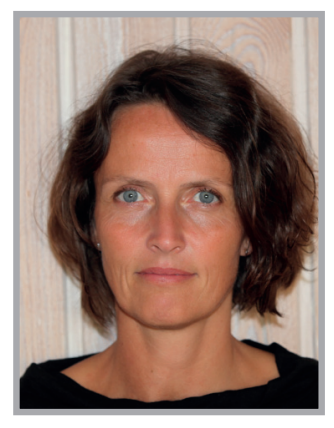

Nanna Finnerup ${ }^{+}$Nanna Finnerup graduated from the Medical School at Aarhus University, Denmark, in 1993, and after an internship worked at the Department of Neurology and Clinical Neurophysiology at the University of Copenhagen, Denmark. Since 1998 she has worked at the Danish Pain Research Center at Aarhus University. She obtained her degree of Doctor of Medical Sciences from Aarhus University in 2008, and is currently Associate Research Professor at the Danish Pain Research Center. Her main research interest is the pathophysiology and therapy of neuropathic pain, with particular focus on the mechanisms of central pain and pharmacological treatment of neuropathic pain. Dr Finnerup is a Management Committee Member of the Special Interest Group on Neuropathic Pain (NeuPSIG) of the International Association for the Study of Pain and a board member of the Scandinavian Association for the Study of Pain (SASP). She is a member of the Editorial Advisory Board of the Scandinavian Journal of Pain, the Encyclopedia of Pain and Pain Management.

Q Have you always been interested in pain research?

After my internship, I was a trainee in neurology, but I've been working as a pain researcher since 1998. My research interest began in clinical neurophysiology in Copenhagen. Together with Professor Anders Fuglsang-Frederiksen, I worked on a standardized computer-based information system for electroencephalography (EEG) as part of the European multicenter project PRESTIGE. When moving to Aarhus in 1998, I started a project on spinal cord injury pain at the Danish Pain Research Center under the supervision of Professor Troels Staehelin Jensen, and I have been interested in pain research ever since. Troels has always been an inspiring mentor and very supportive, and without him I would probably not have chosen a career in pain research. I feel fortunate to have been able to continue my research in pain mechanisms and management because we are still far from fully understanding and providing effective treatment for chronic pain.

\section{Q What drew your attention to researching spinal cord injury pain?}

Spinal cord injury is probably the neurological disease with the highest risk of causing neuropathic pain. 13 years ago, most spinal cord injury research focused on locomotor recovery and bladder and bowel dysfunction, and chronic pain was to a large degree neglected. Much has happened since, and it is now acknowledged that pain is ranked as one of the most disabling consequences of a spinal cord injury. We have been fortunate to work with spinal cord injury rehabilitation experts in Denmark and have collaborators throughout the world with a special interest in spinal cord injury pain. One intriguing research question is why only some of the patients with spinal cord injury develop pain, and our studies of spinal cord patients with and without pain have given us some indications of possible underlying mechanisms. Another important research area for us has been the pharmacological

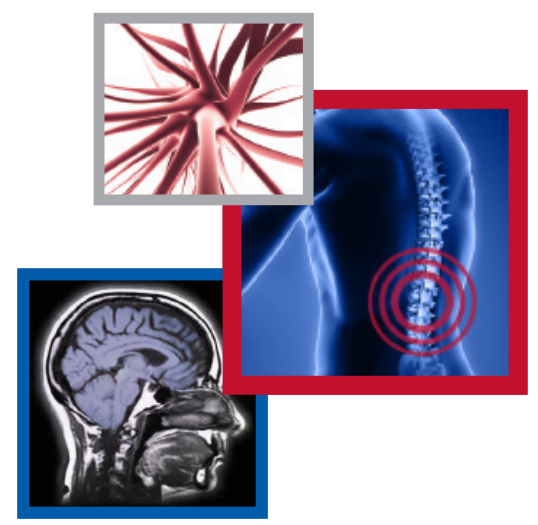

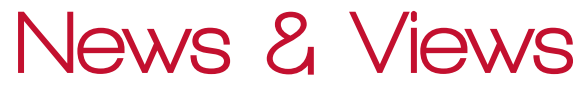

News

Journal Watch

Ask the Experts

Interview

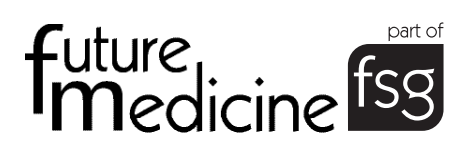


treatment of neuropathic pain in this group of patients, as well as in other chronic pain conditions.

To help advance international standardization of the collection and reporting of pain in spinal cord injury populations, an international spinal cord injury basic standard set has recently been developed, and since 2009 I have been involved in an international group that has worked together on a consensus classification of spinal cord injury pain, which is endorsed by the major spinal cord injury and pain organizations, and hopefully will be published soon.

Q Your main focus is neuropathic pain mechanisms \& evidence-based treatment of neuropathic pain. Could you briefly explain your research on these aspects?

I am still involved in spinal cord injury pain research, with both clinical and experimental studies, but my interest has spread to other types of neuropathic pain as well. We have just finished some studies on neuropathic pain following different plastic surgeries, and some of our current clinical research areas are central poststroke pain and chemotherapy-induced neuropathic pain. Experimental research may also help to elucidate mechanisms of neuropathic pain, and we have been looking into capsaicin-induced sensitization using contact heat evoked potentials.

Over the past couple of decades, the pain field has gained increasing knowledge about neuropathic pain mechanisms; however, treatment is still unsatisfactory. We have followed the publication of pharmacological trials in neuropathic pain, but despite the large increase in randomized controlled trials over the past 5 years, there has been little improvement in the pharmacological treatment of neuropathic pain.

Q According to your opinion \& past studies, what is the role of placebo responses in neuropathic pain trial outcomes \& meta-analyses?

While the use of a placebo group is absolutely essential for evaluating the efficacy of a drug or other pain treatment, it is not without problems. There is currently a large focus on placebo responses and how to minimize them because we have seen an increasing number of negative trials, which may reflect at least some examples of 'study failure' due to very large placebo responses. Studies suggest that the painrelieving effect during the active drug and the placebo is not necessarily additive, and therefore the size of the placebo response affects the estimated drug efficacy. This is a concern in meta-analyses where efficacy is compared across trials with different placebo responses.

Q You are a member of the Neuropathic Pain Special Interest Group (NeuPSIG) of IASP Management Board. What are the aims of this organization?

NeuPSIG is the largest special interest group of IASP with approximately 1300 members. The aim of NeuPSIG is to promote the study of mechanisms, assessment, prevention and treatment of neuropathic pain. Educational activities are important and include reports, surveys, meetings and schools across the world. In February 2012, NeuPSIG will take part in the Annual Congress of the Indian Society for the Study of Pain with two days of educational presentations. Another task of NeuPSIG has been to revise the definition of neuropathic pain, and the most recent redefinition is now accepted by IASP. Currently, we are reviewing current classifications of neuropathic pain, which is the starting point for the NeuPSIG Subcommittee on Classification, which will discuss a new classification of neuropathic pain.

\section{Q What are your plans for further research \& study?}

I hope to be able to continue my clinical research in neuropathic pain, but also to be involved in experimental pain models and preclinical studies. We are part of the 'Europain' Investigational Medicines Initiative, and one of the main projects that we have been able to initiate is a large randomized trial together with Odense University Hospital focusing on predictors of response based on pain phenotypes rather than etiology. A mechanism-based approach to pain treatment is heavily discussed, and many initiatives have been 
published to classify patients according to quantitative sensory testing profiles or pain descriptors. However, it remains to be shown whether such classifications reflect different pathophysiological mechanisms and whether they will have treatment implications. We also plan to further study mechanisms of specific neuropathic pain symptoms; for example, cold allodynia, which is seen commonly in central pain and certain types of chemotherapy-induced pain.

Q How would you like to see the field of neuropathic pain management/research advance in the next few years?

Many challenges remain in neuropathic pain research. First and foremost, we need to be able to treat neuropathic pain better. While we have different treatment options, there are patients who do not benefit sufficiently or not at all from these treatments, even when used in combinations, or do not tolerate the treatments. The study of mechanism-based individualized treatments and the study of risk factors and genetic and psychosocial factors and their relation to pain are of great importance, as we need to further improve our understanding of how the pain transmission system works and changes upon injury to develop new and better intervention strategies and treatment options. At present, neuropathic pain is not a condition we know how to prevent once there has been an injury to the somatosensory nervous system. A cascade of events follows the injury leading to sensitization and spontaneous activity of the pain pathways. We are beginning to understand many of the mechanisms involved, but to prevent these maladaptive changes from happening or to selectively silence the pain system is a major challenge.

\section{Financial \& competing}

interests disclosure

$N$ Finnerup receives research funding from the Europain Investigational Medicines Initiative, which is a public-private partnership between the pharmaceutical industry and the EU. N Finnerup has also received research funding from Grünenthal and served as consultant for Grünenthal and Pfizer. $N$ Finnerup has no other relevant affiliations or financial involvement with any organization or entity with a financial interest in or financial conflict with the subject matter or materials discussed in the manuscript apart from those disclosed.

No writing assistance was utilized in the production of this manuscript. 